\title{
CONHECIMENTO ESPECIALIZADO DE PROFESSORES DE QUÍMICA: MODELO TEÓRICO
}

\author{
CHEMISTRY TEACHER'S SPECIALIZED KNOWLEDGE: THEORETICAL \\ MODEL
}

\author{
Susel Tais Soares ${ }^{1}$ \\ ORCID iD: $\underline{0000-0003-4681-968 X}$ \\ Stela Silva Lima \\ ORCID iD: $\underline{0000-0002-6743-8969}$ \\ Leandro Carbo ${ }^{3}$ \\ ORCID iD: $\underline{0000-0001-5514-7040}$
}

\begin{abstract}
RESUMO
A proposta de modelos educacionais tem sido objeto de estudo nas últimas décadas; a área da Matemática tem recebido maior destaque em comparação com as demais, inclusive possui o modelo teórico denominado: Conhecimento Especializado de Professores de Matemática - MTSK. Este modelo possibilita a descrição do conjunto de conhecimentos matemáticos necessários para o ensino e a aprendizagem de determinado conteúdo matemático. Embora a Matemática tenha sido pioneira no modelo de Conhecimento Especializado de Professores e existam outros modelos genéricos para a área das ciências da natureza, somente em 2018 a física propôs um modelo teórico por meio da transposição do MTSK. Dessa forma, o objetivo desta pesquisa foi propor um modelo teórico para a área da química, por intermédio da transposição do MTSK, com intuito de diminuir a desconexão do conhecimento científico com o pedagógico, permitir que o docente possa aprimorar seus conhecimentos para ensinar química e, consequentemente, contribuir para os processos de ensino e aprendizagem. A pesquisa seguiu a mesma metodologia aplicada para a área da física, através da transposição do MTSK resultando no modelo Conhecimento Especializado de Professores de Química - CTSK. O resultado foi satisfatório, uma vez que atendeu aos objetivos propostos, resultando no CTSK, um modelo teórico com dois domínios, um referente ao Conhecimento Químico e outro ao Conhecimento Didático do Conteúdo; cada domínio possui três subdomínios, e; o modelo ainda considera as crenças provenientes, tanto dos professores quanto dos alunos.
\end{abstract}

\footnotetext{
${ }^{1}$ Mestra em Ensino, Instituto Federal de Educação, Ciência e Tecnologia de Mato Grosso (IFMT). Professora no Instituto Federal de Educação, Ciência e Tecnologia de Mato Grosso (IFMT), Cuiabá, Mato Grosso, Brasil. Endereço para correspondência: Av. Juliano Costa Marques, s/n, Bela Vista, Cuiabá, Mato Grosso, Brasil, CEP: 78.050-560. E-mail: susel.soares@blv.ifmt.edu.br.

${ }^{2}$ Mestra em Ensino, Instituto Federal de Educação, Ciência e Tecnologia de Mato Grosso (IFMT). Professora no Instituto Federal de Educação, Ciência e Tecnologia de Mato Grosso (IFMT), Cuiabá, Mato Grosso, Brasil. Endereço para correspondência: Rua Profa. Zulmira Canavarros, 95, Centro, Cuiabá, Mato Grosso, Brasil, CEP: 78005-200. E-mail: stela.lima@ cba.ifmt.edu.br.

${ }^{3}$ Doutor em Química, Universidade Estadual Paulista (UNESP). Professor no Instituto Federal de Educação, Ciência e Tecnologia de Mato Grosso (IFMT), Campo Verde, Mato Grosso, Brasil. Endereço para correspondência: Rodovia BR-364, Km 329, s/n, Campo Verde, Mato Grosso, Brasil, CEP: 78106-970. E-mail: leandro.carbo@svc.ifmt.edu.br.
} 
Palavras-chave: Ensino de Química. CTSK. MTSK. Conhecimento Especializado de Professores. PCK.

\begin{abstract}
The proposal of educational models has been object of study in the last decades, the area of the mathematics has received greater prominence in comparison with the others, also owns the theoretical model denominated Mathematics Teacher's Specialized Knowledge - MTSK. This model allows the description of the set of mathematical knowledge necessary for the teaching and learning of a certain mathematical topic. Although mathematics was a pioneer at the model of Teacher`s Specialized Knowledge and also there are other generic models for the area of the natural sciences, only in 2018, physics proposed the model through the transposition of the MTSK. Thus, the objective of this research was to propose a theoretical model Teacher`s Specialized Knowledge for the area of chemistry, through the transposition of the MTSK, in order to reduce the disconnection of scientific chemistry and pedagogical knowledge, to allow teachers to improve their knowledge to teach chemistry, consequently contributing to the teaching-learning process. The research followed the same methodology applied to the field of physics, through the transposition of the MTSK resulting in the theoretical model Chemistry Teacher`s Specialized Knowledge - CTSK. The result was satisfactory, since it met the proposed objectives resulting in the CTSK, a theoretical model with two domains, one referring to Chemistry Knowledge and another to Pedagogical Content Knowledge, each domain has three subdomains and the model still considers the beliefs coming from both teachers and students.
\end{abstract}

Keywords/Palabras clave: Chemistry Teaching. CTSK. MTSK. Teacher's Specialized Knowledge. PCK.

\title{
1 INTRODUÇÃO
}

A química é uma disciplina relativamente nova; antigamente seus conhecimentos eram desenvolvidos como parte dos conteúdos da física, inclusive os primeiros diplomas da área emitidos no país, há pouco mais de cem anos; começou com nível técnico, ofertado pelo Mackenzie College, que logo se tornou um curso de nível superior e isso ocorreu no mesmo ano, 1919, em que foi inaugurada a Escola Superior de Química da Escola Oswaldo Cruz (SILVA; SCHNETZLER, 2005; LIMA, 2018).

No ensino de química há problemas tanto quanto em qualquer outro setor do segmento educacional; essa problemática está relacionada com a complexidade da disciplina (QUADROS et al., 2011), bem como a formação docente ineficaz, ocasionada pela desconexão do conhecimento da química com o pedagógico, um fator importante e citado por alguns autores em diferentes contextos (LOPES, 1997; GARCIA, 2009; OLIVEIRA; REZENDE, 2011).

Fazendo-se uma breve retrospectiva da evolução educacional nos últimos trinta anos, que visa contribuir com a conexão destes conhecimentos, da disciplina e o pedagógico, pode-se partir do modelo educacional proposto na década de 80 por Shulman (1986), Pedagogical Content Knowledge - PCK, Conhecimento Pedagógico do Conteúdo, que foi citado significativamente por pesquisadores, segundo os dados apresentados nas ferramentas acadêmicas, e que contribuiu 
especificamente para a evolução da pesquisa educacional, principalmente na área da Matemática (GOES, 2014).

Tendo em vista o destaque da Matemática em pesquisas educacionais, em 2008 houve uma proposta de um modelo teórico relevante: Mathematics Knowledge for Teaching - MKT, Conhecimento Matemático para o Ensino (BALL; THAMES; PHELPS, 2008), mas que apresentou algumas lacunas o que, posteriormente, possibilitou a proposta de um novo modelo teórico, denominado: Mathematics Teacher's Specialized Knowledge - MTSK, Conhecimento Especializado de Professores de Matemática (CARRILLO et al., 2014). Atualmente o MTSK tem tido repercussão mundial, como por exemplo, em Portugal está em desenvolvimento um projeto de pesquisa para obtenção do título de doutor na área de Biologia (LUÍS; MONTEIRO; CARRILLO, 2015).

No Brasil alguns estudos vêm sendo realizados a partir do MTSK para área da matemática (MORIEL JUNIOR, 2014; MORIEL JUNIOR; WIELEWSKI, 2017; VASCO; MORIEL JUNIOR; CONTRERAS, 2017), para a área da física (LIMA et al., 2017; LIMA, 2018), para a área da Biologia (MARQUES; MORIEL JUNIOR, 2019; MARQUES; MORIEL JUNIOR, 2020) e também para química (SOARES, 2019; SOARES et al., 2019). Além da Espanha, Portugal e Brasil, outros países como Chile, México, Colombia, Equador e Peru também desenvolvem pesquisa com, ou a partir do, MTSK.

A partir do cenário abordado, as problemáticas na área do ensino de química e a proposta de modelos teóricos que têm ocorrido nos últimos 35 anos, faz-se necessário atualizar o ensino de química com a proposta de um modelo que conecta o conhecimento específico ao didático para a área. Dessa forma, a problemática da presente pesquisa está no questionamento de quais são as características do modelo teórico do Conhecimento Especializado de Professores de Química - Chemistry Teacher's Specialized Knowledge - CTSK, tendo-se por referência a base conceitual do modelo do Conhecimento Especializado de Professores de Matemática - MTSK.

A justificativa da pesquisa tem três vieses, sendo o primeiro ser uma pesquisa pioneira para a área química, apesar de existirem modelos a partir do PCK para a área da química, não havia ainda a proposta do conhecimento especializado. Alguns modelos se referiam à área de ciências da natureza, outros englobavam ciências e matemática de modo geral, e os modelos relativos à área química estabeleciam hierarquia entre conhecimentos. Dessa forma, teve-se a iniciativa de partir de um modelo especializado da área da matemática para a química, propondo um modelo especializado para a área, que possibilita a conexão do conhecimento químico com o conhecimento didático para o ensino de química. O segundo viés quanto ao vínculo da pesquisa 
ao TSK Group - Teacher's Specialized Knowlegde Research Group ${ }^{4}$ Grupo de Pesquisa do Conhecimento Especializado de Professores do Instituto Federal de Educação, Ciência e Tecnologia de Mato Grosso - IFMT que atua com pesquisas voltadas ao conhecimento e formação docente para o ensino de matemática e ciências naturais, campo este que possui grande demanda de pesquisas acadêmicas, uma vez que está relacionado ao processo de ensino de matemática e ciências, neste caso, especificamente ao ensino de química. O TSK Group está vinculado à Red Iberoamericana $M T S K^{5}$ que propôs o modelo teórico de Conhecimento Especializado de Professores de Matemática e, atualmente, possui núcleos de trabalhos em diversos países, tais como: Espanha, Portugal, Chile, Equador, Peru, México e Brasil, coordenado pelo Professor Dr. José Carrillo Yáñez.

O terceiro viés se baseia no fato da literatura apontar que o conhecimento especializado permite ao professor aprimorar seus conhecimentos para ensinar, o que é importante para a área da química, uma vez que o conhecimento especializado propicia um nível de aprofundamento, organização e estruturação superior ao domínio da matéria a ensinar (CARRILLO YÁÑ̃E; GONZÁLEZ; NAVARRO, 2015), algo que pode vir a possibilitar a valorização profissional perante a sociedade, além das contribuições para o processo educacional.

Dessa forma, o objetivo do trabalho foi propor um modelo teórico capaz de descrever o conjunto de Conhecimento Especializado de Professores de Química-CTSK, a partir da análise dos domínios e subdomínios do MTSK.

\section{REFERENCIAL TEÓRICO}

\subsection{O Ensino de Química}

A química, apesar de ser direcionada ao entendimento de fenômenos cotidianos, é uma disciplina bastante complexa e os alunos possuem dificuldades para compreendê-la, fato este comprovado pelo resultado dos exames oficiais, como ENEM, vestibulares, dentre outros (QUADROS et al., 2011).

$\mathrm{O}$ apontamento literário mais frequente quanto à problemática do processo de ensino de química remete ao fato de o conhecimento químico específico estar totalmente desconectado do conhecimento pedagógico. Embora haja pesquisas identificando os problemas, propondo

\footnotetext{
4 Grupo de Pesquisa do Conhecimento Especializado de Professores do IFMT (http://dgp.cnpq.br/dgp/espelhogrupo/529979).

${ }^{5}$ http://www.redmtsk.com/
} 
técnicas, métodos, sugestões de abordagem de determinado conteúdo, estabelecendo qual é o conhecimento químico necessário, elas não conectam com o conhecimento pedagógico, ou seja, tem-se uma valorização do conhecimento químico, porém uma desvalorização do conhecimento sobre o ensino (GARCIA, 2009; OLIVEIRA; REZENDE, 2011).

Em outros momentos, o foco está basicamente na parte pedagógica e isso influencia, questionando a qualidade da formação docente (GARCIA, 2009). Em trabalho realizado por Oliveira e Rezende (2011) houve um relato por parte de um licenciando sobre a desarticulação entre disciplinas pedagógicas e conteúdo específico nas universidades brasileiras em cursos de licenciatura, indicando que há um problema significante na formação de professores de química para atuação na área educacional.

Há pesquisadores que indagam a falta de suporte capaz de ordenar o conhecimento científico, investigativo e prático; inclusive sugerem a criação de um quadro teórico e conceitual que possa auxiliar desde o planejamento, o processo de desenvolvimento, até a avaliação. Entretanto, em nenhum momento citam a necessidade do conhecimento pedagógico e, depois, remetem tal problemática ao fato da química ser uma área relativamente nova, principalmente em termo brasileiro (SILVA; SCHNETZLER, 2005).

Lopes (1997) afirma que é função do professor ensinar não somente o conteúdo químico específico, mas também todo o conhecimento integrado às exigências didáticas, pois a didática é mais que a adaptação do conhecimento científico, deve estar vinculada ao processo de produção educacional e, para isso, faz-se necessário integrar ambos os conhecimentos, específico e pedagógico.

Alguns autores abordam a existência de materiais didáticos desatualizados, ou mesmo o fato de serem limitados, não contemplando determinados conteúdos, assuntos, teorias e/ou modelos; problemas da abordagem descontextualizada dos conteúdos; alta complexidade da disciplina, talvez devido à linguagem química que envolve símbolos, equações, reações, modelos, fórmulas estruturais, ou mesmo, gráficos; e o fato da química possuir um currículo que a literatura determina como sobrecarregado (LOPES, 1997; SCHNETZLER, 1992; LOPES, 2005; ROQUE; SILVA, 2008; SILVA, 2012; MASSENA et al., 2013; MELO; NETO, 2013; CORREIA et al., 2015).

Todas essas questões podem ocasionar uma problemática em determinar o que selecionar para ensinar e como ensinar, tudo isso justificando a necessidade de melhorar o processo de ensino nesta área, talvez não só pela falta de conexão entre o conhecimento específico e o pedagógico, como também por todos os inconvenientes supracitados que também são frutos desta desconexão (LOPES, 1997; SILVA; SCHNETZLER, 2005; GARCIA, 2009; OLIVEIRA; 
REZENDE, 2011).

\subsection{Modelos de Ensino}

No processo educacional existem dois importantes personagens: o professor e o aluno; o primeiro atua como mediador, orientador, motivador, facilitador e responsável por estabelecer um planejamento para que seja aplicado em sala de aula determinado conteúdo, enquanto o segundo tem o papel de investigar, confrontar, explorar e ampliar seus conhecimentos (PETRUCCI; SCHNETZER, 1998), porém, nenhum dos dois papéis são pré-estabelecidos.

Ambos os personagens contribuem com suas crenças nestes processos de ensino e de aprendizagem. Para a disciplina de química, o aluno vem com suas concepções prévias sobre os fenômenos naturais, que entram em conflito com os conhecimentos científicos, propiciando um processo de mudança conceitual, proporcionada pelo processo de ensino. Enquanto o professor, por meio de suas práticas pedagógicas, demonstra não somente seus conhecimentos, mas também suas crenças, o que leva a literatura a criticar a formação docente do modelo tradicional, apontando a necessidade de melhoria (SCHNETZLER, 2002).

A partir da década de 80, a educação começou a avançar devido à publicação de Shulman (1986) sobre o Pedagogical Content Knowledge - Conhecimento Pedagógico do Conteúdo (PCK). Este autor é reconhecido mundialmente devido ao PCK; possui atualmente mais de noventa e cinco mil citações dos seus trabalhos, segundo seu perfil no Google Acadêmico, em pesquisa realizada em abril de 2020, e tem sido base para inúmeros estudos de diversas áreas educacionais.

O PCK é um modelo geral que contribui para determinar o conhecimento profissional em diversas áreas, considerando aspectos educacionais que possam contribuir para a aprendizagem dos alunos, por intermédio de sete categorias base do conhecimento (SHULMAN, 1987).

Com o decorrer dos anos, vários pesquisadores têm contribuído com modelos sobre o PCK; dentre os modelos relacionados, têm-se os referentes ao conhecimento básico para o professor, que parte do modelo de Shulman, seguindo com o Modelo de Tamir; Modelo de Grossman; Modelo de Koballa, Gräber, Coleman e Kemp; Modelo de Morine-Dershimer e Kent; Modelo de Carlsen; Modelo de Barnett e Hodson; Modelo de Banks, Leach e Moon; Modelo de Hashweh, e; Modelo da Cúpula do PCK; e outros referentes ao Conhecimento Pedagógico do Conteúdo: Modelo de Marks; Modelo de Cochran, De Ruiter e King; Modelo de FernandezBalboa e Stiehl; Modelo de Geddis e Wood; Modelo de Magnusson, Krajcik e Borko; Modelo de Veal e Makinster; Modelo de Rollnick, Rhemtula, Dharsey e Ndlovu, e; Modelo de Park e 
Oliver (GOES, 2014), como a linha do tempo apresentada na Figura 01.

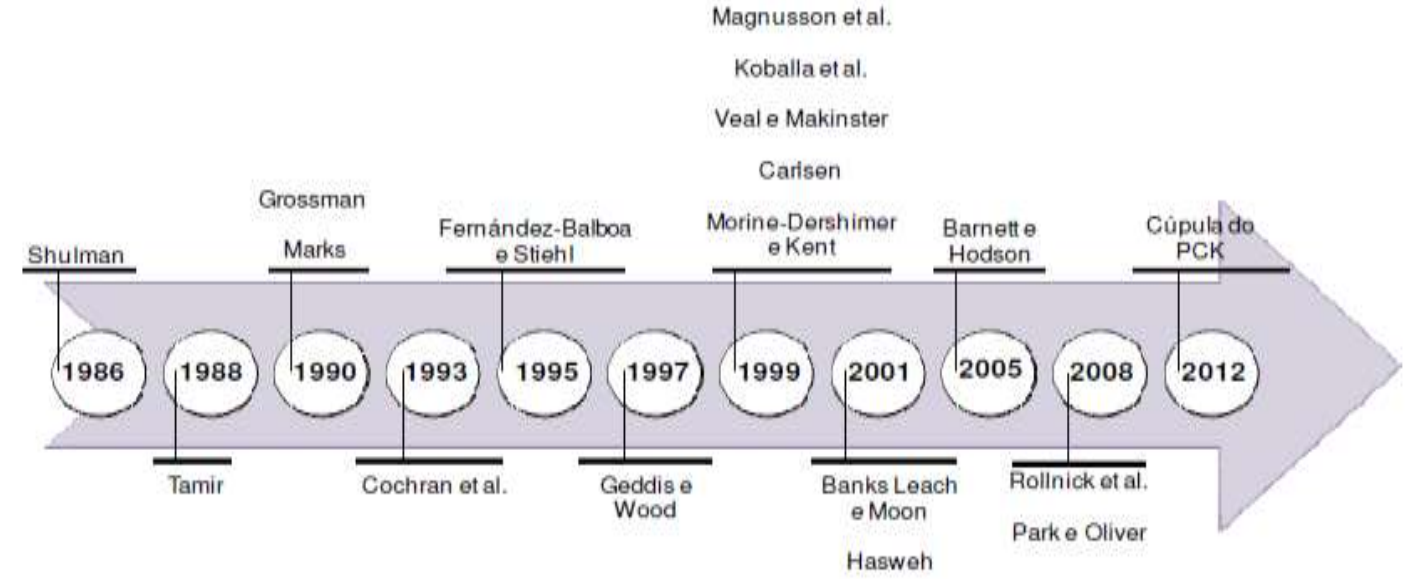

Figura 1 - Linha do tempo de modelos relacionados ao PCK. Fonte: Goes (2014, p. 38).

O modelo proposto por Park e Oliver, em 2008, destaca-se devido à utilização de conhecimentos químicos no seu desenvolvimento, entretanto foi elaborado para contemplar a área de ciências como um todo, e devido a sua proposta em formato hexagonal, mesmo aspecto proposto no modelo desenvolvido por Carrillo (2014) e seus colaboradores, que é o modelo teórico matemático titulado Conhecimento Especializado de Professores de Matemática, Mathematics Teacher's Specialized Knowledge - MTSK. Ambas características foram importantes para o desenvolvimento desta pesquisa.

Embora grande parte das produções acadêmicas seja dos Estados Unidos, a literatura aponta a existência de um grupo de pesquisa brasileiro da Universidade de São Paulo que tem contribuído com estudos do PCK, voltado para a área das ciências da natureza e formação docente (FERNANDEZ, 2015).

O PCK tem sido objeto de estudo em diversas áreas, inclusive na química. Um estudo recente (GOES, 2014) trouxe um levantamento dos trabalhos realizados no período de 1986 a 2013, que apontou 154 trabalhos empíricos na área, um resultado significativo, porém não tanto quanto a matemática, que obteve o maior destaque, com 739 trabalhos. Na área das ciências da natureza e matemática, a contribuição em ordem decrescente foi matemática, ciências, química, física, biologia e ambiental, e astronomia, respectivamente.

O destaque da matemática nas produções se justifica pelo fato desta área se encontrar em estado mais avançado de pesquisa que as demais e, inclusive, possuir o modelo referência para este trabalho, o Conhecimento Especializado de Professores de Matemática. 


\subsection{Conhecimento Especializado de Professores de Matemática}

O modelo teórico matemático, capaz de descrever o Conhecimento Especializado de Professores de Matemática - MTSK, foi desenvolvido pela Rede Iberoamericana, coordenado pelo Prof. Dr. José Carrillo Yañez e seus colaboradores, em 2014, a partir dos modelos: PCK de Shulman (1986), que se trata de um modelo genérico, aplicado em diversas áreas e engloba vários cenários, e do modelo Conhecimento Matemático para o Ensino, Mathematics Knowledge for Teaching - MTK (BALL; THAMES; PHELPS, 2008).

O MTK foi um progresso científico para a área da matemática, uma vez que envolve conhecimento e habilidades matemáticas, porém com lacunas, por não reconhecer o conhecimento didático do conteúdo e devido aos conflitos na comparação com outros subdomínios (MONTES; CONTRERAS-GONZALEZ; CARRILLO, 2013; MORIEL JUNIOR, 2014; CARRILLO YÁÑEZ; GONZÁLEZ; NAVARRO, 2015; CARRILLO YAÑEZ et al., 2018).

O MTSK é um modelo teórico que estabelece o conjunto de conhecimentos especializados fundamentais para o ensino de matemática (CARRILLO et al., 2014; MORIEL JUNIOR; WIELEWSKI, 2017); foi desenvolvido visando contemplar, resumidamente, os conhecimentos relativos: às definições e conceitos matemáticos; às conexões entre os conhecimentos matemáticos; à prática matemática; aos conhecimentos relativos à estratégia de ensino de matemática; ao processo de aprendizagem matemática; e ao currículo (CARRILLO YAÑEZ et al., 2014; CARRILLO YAÑEZ et al., 2018).

Com o objetivo de propor um modelo de conhecimento especializado para a matemática, os autores fizeram um estudo bibliográfico com o intuito de propor um MTSK inicial; depois, buscaram lacunas relativas a conhecimentos que não haviam sido contemplados por meio de testes empíricos, como aplicação do modelo em episódios de ensino, cursos de formação de professores, entrevistas com profissionais atuantes desde o Ensino Básico ao Superior, para então propor o modelo final, descrevendo os conhecimentos relativos a cada subdomínio pertencente ao MTSK (CARRILLO YAÑEZ et al., 2018).

Dessa forma, o MTSK possui um conjunto de dois domínios: Conhecimento Matemático, Mathematics Knowledge - MK e Conhecimento Didático do Conteúdo, Pedagogical Content Knowledge - PCK (tradução própria); e seis subdomínios, sendo três em cada domínio, conforme pode ser observado na Figura 02. 


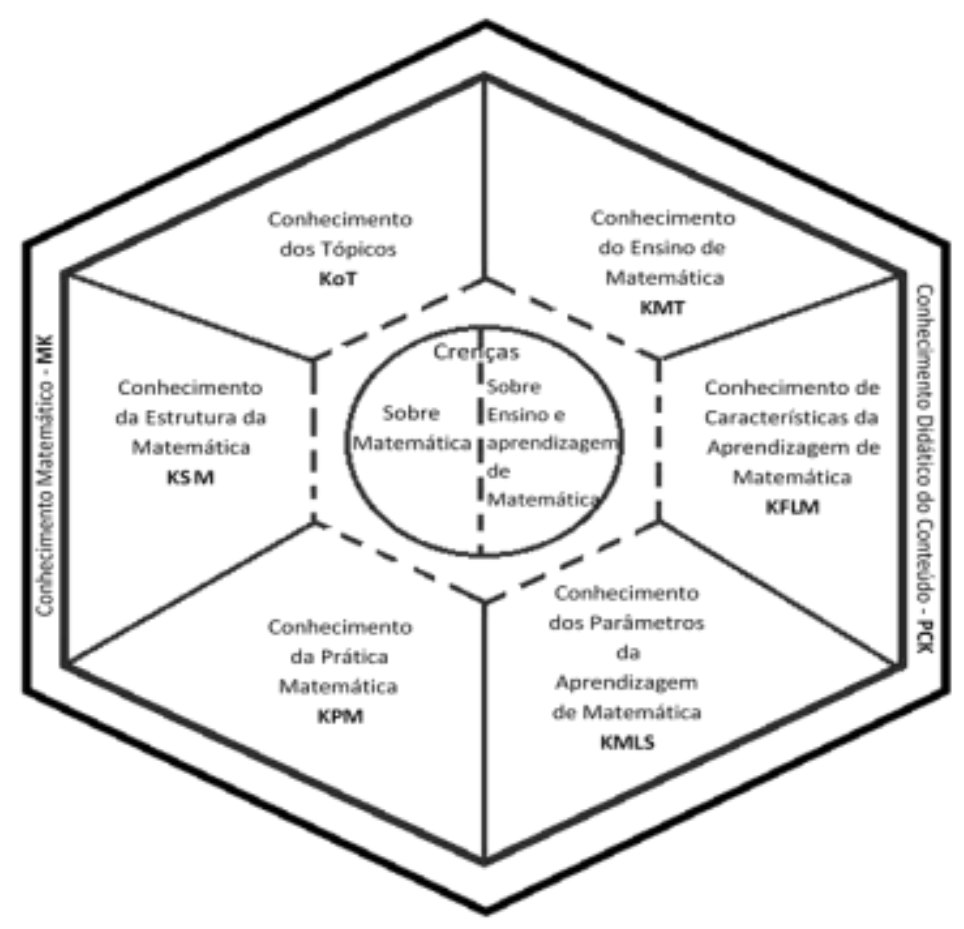

Figura 2 - Modelo MTSK.

Fonte: Carrillo et al. (2014, tradução nossa).

No domínio do Conhecimento Matemático, tem-se os subdomínios: Conhecimento de Tópicos Matemáticos, Knowledge of Topics - KoT contempla conhecimentos relativos a definições, propriedades, conceitos, teoremas, próprio do conteúdo matemático; Conhecimento da Estrutura da Matemática, Knowledge of the Structure of Mathematics - KSM que engloba as conexões existentes entre conceitos matemáticos, e; Conhecimento da Prática Matemática, Knowledge of Practices in Mathematics - KPM que abarca conhecimentos relativos a criação, dedução e desenvolvimento da matemática, ou seja, conhecimentos capazes de argumentar para criar novos conhecimentos matemáticos por meio de exemplos e contraexemplos (CARRILLO YAÑEZ et al., 2018; CARRILLO et al., 2014; MORIEL JUNIOR, 2014; VASCO; MORIEL JUNIOR; CONTRERAS, 2017) (tradução própria).

No domínio Conhecimento Didático do Conteúdo tem-se os subdomínios: Conhecimento do Ensino de Matemática, Knowledge of Mathematics Teaching - KMT que contempla conhecimentos relativos a estratégias de ensino, recursos didáticos e teorias de ensino; Conhecimento das Características de Aprendizagem de Matemática, Knowledge of Features of Learning Mathematics - KFLM que contempla conhecimentos relativos à teoria de aprendizagem, assimilação, erros, dificuldades, formas de interação com um conteúdo matemático, interesses e expectativas, e; o Conhecimento dos Parâmetros da Aprendizagem de Matemática, Knowledge of Mathematics Learning Standards - KMLS que abrange 
conhecimentos relativos a metas, sequência dos conteúdos e expectativa de aprendizagem. (CARRILLO YAÑEZ et al., 2018; CARRILLO et al., 2014; MORIEL JUNIOR, 2014) compondo um modelo especializado para a área (tradução própria).

Exceto o subdomínio KPM, cada um dos demais, pertencentes ao MTSK, possuem categorias associadas aos conhecimentos referenciados acima, conforme pode ser verificado na Tabela 01. Embora esta definição estivesse sido estabelecida desde 2016 em documento interno, somente recentemente foi publicada (CARRILLO YANEZ et al., 2018; Seminario de Investigación en Didáctica de la Matemática - SIDM, 2016).

Tabela 1 - Modelo MTSK com definição de subdomínios e categorias

\begin{tabular}{|c|c|c|}
\hline Domínios & Subdomínios & Categorias \\
\hline \multirow{3}{*}{$\begin{array}{l}\text { Conhecimento } \\
\text { Matemático }\end{array}$} & $\begin{array}{l}\text { KoT - Conhecimento de } \\
\text { Tópicos Matemáticos }\end{array}$ & $\begin{array}{l}\text { 1. Procedimentos (Como fazer? Quando fazer? } \\
\text { Por que é feito por este caminho? } \\
\text { Características do resultado) } \\
\text { 2. Definições, propriedades e fundamentos } \\
\text { 3. Registro de representação } \\
\text { 4. Fenomenologia e aplicações }\end{array}$ \\
\hline & $\begin{array}{l}\text { KSM - Conhecimento } \\
\text { da Estrutura da } \\
\text { Matemática }\end{array}$ & $\begin{array}{l}\text { 1. Conexões baseadas na simplificação } \\
\text { 2. Conexões baseadas na complexidade } \\
\text { 3. Conexões auxiliares } \\
\text { 4. Conexões transversais }\end{array}$ \\
\hline & $\begin{array}{l}\text { KPM - Conhecimento } \\
\text { da Prática Matemática }\end{array}$ & \\
\hline \multirow{3}{*}{$\begin{array}{l}\text { Conhecimento } \\
\text { Didático } \\
\text { do } \\
\text { Conteúdo }\end{array}$} & $\begin{array}{l}\text { KMT - Conhecimento } \\
\text { do Ensino de } \\
\text { Matemática }\end{array}$ & $\begin{array}{l}\text { 1. Teorias de ensinar matemática } \\
\text { 2. Recursos didáticos (físicos e digitais) } \\
\text { 3. Estratégias, técnicas, tarefas e exemplos }\end{array}$ \\
\hline & $\begin{array}{l}\text { KFLM - Conhecimento } \\
\text { das Características de } \\
\text { Aprendizagem de } \\
\text { Matemática }\end{array}$ & $\begin{array}{l}\text { 1. Teorias de aprendizagem matemática } \\
\text { 2. Pontos fortes e dificuldades em aprender } \\
\text { matemática } \\
\text { 3. Formas de interação dos alunos com um } \\
\text { conteúdo matemático } \\
\text { 4. Perspectiva de interesse de aprender } \\
\text { matemática }\end{array}$ \\
\hline & $\begin{array}{l}\text { KMLS - Conhecimento } \\
\text { dos Parâmetros da } \\
\text { Aprendizagem de } \\
\text { Matemática }\end{array}$ & $\begin{array}{l}\text { 1. Expectativa de aprendizagem } \\
\text { 2. Expectativa do nível de desenvolvimento } \\
\text { conceitual ou procedimental } \\
\text { 3. Sequência dos tópicos }\end{array}$ \\
\hline
\end{tabular}

São consideradas ainda no modelo MTSK as Crenças sobre Matemática, pertencente ao domínio Conhecimento Matemático, e sobre Ensino e Aprendizagem de Matemática, pertencente ao domínio Conhecimento Didático do Conteúdo, que se encontram interligadas a todos os subdomínios do respectivo domínio (Figura 02). As crenças são provenientes da experiência 
profissional e pessoal do professor e, independentemente do grau, influencia no processo educacional, embora seja uma convicção do docente e não um conhecimento fundamentado cientificamente (CARRILLO et al., 2014).

A literatura aponta que o conhecimento especializado permite ao docente aprimorar seus conhecimentos para ensinar e possibilita diminuir as diferenciações de rendimento escolar em diferentes colégios, uma vez que o conhecimento especializado propicia um nível de aprofundamento, organização e estruturação superior ao domínio da matéria a ensinar (CARRILLO YÁÑEZ; GONZÁLEZ; NAVARRO, 2015).

Segundo Góes (2014), para Shulman, o professor, para ser um profissional especializado, não deve apenas refletir sobre a prática de ensinar, mas agir, de modo a destacar suas habilidades e seus conhecimentos didáticos.

\section{METODOLOGIA}

A investigação está classificada como qualitativa (BOGDAN; BIKLEN, 1994) e exploratória-descritiva (GIL, 2002), a mesma metodologia encontradas nos trabalhos do grupo de pesquisa aplicados às áreas da física e biologia através da transposição do MTSK para a área da química, resultado na proposta de um modelo teórico capaz de descrever os Conhecimentos Especializados de Professores de Química - CTSK (LIMA, 2018; SOARES, 2019; SOARES et al., 2019; MARQUES; MORIEL, 2019; MARQUES; MORIEL JUNIOR, 2020).

\section{ANÁLISES E RESULTADOS}

Foi realizada a transposição direta do MTSK para a área da química resultando na proposta do modelo teórico para a área de ensino de química, o qual possibilita descrever o conjunto de conhecimentos especializados de professores da área. O modelo foi nomeado como Conhecimento Especializado de Professores de Química (Chemistry Teacher`s Specialized Knowledge - CTSK). A proposta do nome e a sigla foram em inglês para padronização internacional. Tal estratégia foi adotada tanto para nomeação do modelo, como nas divisões estabelecidas dentro do mesmo, por meio de domínios e subdomínios, mantendo o mesmo procedimento adotado tanto no modelo teórico matemático denominado MTSK, como na área química que possui outras padronizações internacionais, como: nomenclaturas, símbolos dos elementos químicos, entre outros.

O CTSK possui formato hexagonal, originado da transposição, está dividido em dois domínios: Conhecimento Químico (Chemistry Knowledge - CK) e Conhecimento Didático do 
Conteúdo (Pedagogical Content Knowledge - PCK). Cada domínio está dividido em três subdomínios e modelo ainda considera as crenças Sobre Química e Sobre Ensino e Aprendizagem de Química (Figura 1).

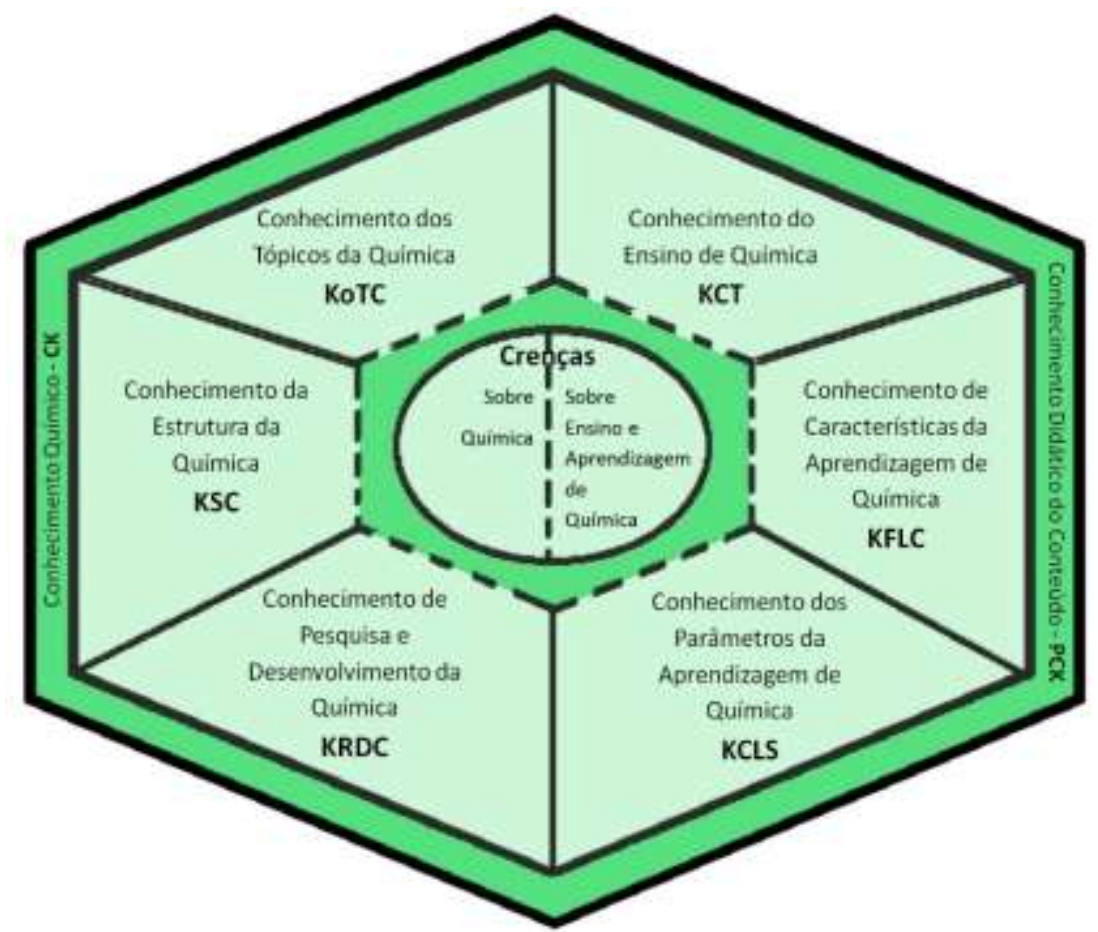

Figura 1 - Modelo teórico Conhecimento Especializado de Professores de Química Fonte: Soares (2019, p. 77, adaptação).

\subsection{Domínio: Conhecimento Químico (Chemistry Knowledge - CK)}

Este domínio engloba o conjunto de conhecimentos químicos referentes à química pura, que permite ao docente distinguir o conjunto de conhecimentos adequados ao processo de ensino e de aprendizagem sobre um determinado tema.

Ele possui três subdomínios, sendo eles: Conhecimento dos Tópicos da Química (Knowledge of Topics of Chemistry - KoTC); o Conhecimento da Estrutura da Química (Knowledge of the Structure of Chemistry - KSC); e Conhecimento de Pesquisa e Desenvolvimento da Química (Knowledge of Research and Development of Chemistry - KRDC).

\section{Conhecimento dos Tópicos da Química - KoTC}

O subdomínio do Conhecimento dos Tópicos da Química engloba conhecimentos químicos relacionados aos conteúdos da química pura, envolvendo desde modelos químicos, 
teorias, leis, conceitos, definições, fórmulas químicas, gráficos, representações até conhecimento de experimentos teóricos e aplicações, todos próprios dos conteúdos químicos ou referente a conhecimento de conteúdos relativos a outras disciplinas que são necessários à compreensão dos conhecimentos químicos.

As representações na área referem-se às fórmulas moleculares, geométricas, empírica, estrutural planar, estrutura de Lewis, estrutura de Kekulé, estrutura de linhas, elementos químicos, ligações químicas, grupos funcionais, gráficos, representação de reações, mecanismos, modelos químicos, grandezas, etc.

Ainda faz parte deste subdomínio conhecimentos relativos à história da química e conhecimentos populares.

\section{Conhecimento da Estrutura da Química - KSC}

Neste subdomínio, os conhecimentos químicos são relativos à estrutura da química e suas conexões dentro da própria disciplina e com conhecimentos de disciplinas correlatas, como a matemática, física e biologia.

Estas conexões podem ocorrer quando: há diferença em níveis de avanço entre determinados conteúdos; simplifica determinado conteúdo para ensinar; possui características em comum; determinado conhecimento pode ser utilizado para auxiliar a construção de um novo conhecimento, incluindo a conexão com conhecimentos relativos à interdisciplinaridade da química com matemática, física e biologia; determinado conhecimento teórico é utilizado para construção de um conhecimento prático e vice-versa; há uma aplicabilidade correlata e; determinada representação da linguagem química conecta-se com determinado conteúdo teórico, etc.

\section{Conhecimento de Pesquisa e Desenvolvimento da Química - KRDC}

Refere-se ao desenvolvimento investigativo da química para gerar novos conhecimentos referentes à química pura, englobando conhecimentos relativos a deduções químicas; desenvolvimento de metodologias teóricas/experimentais relativas à química pura; pesquisa investigativa e desenvolvimento argumentativo com exemplos e contraexemplos, sejam teóricos e/ou experimentais; pesquisa relativa ao levantamento de hipóteses; e desenvolvimento de processos. 
Os conhecimentos relativos à pesquisa e ao desenvolvimento da química, considerados neste subdomínio, podem ser tanto teóricos quanto experimentais.

\subsection{Conhecimento Didático do Conteúdo - PCK}

Este domínio engloba o conjunto de conhecimentos didáticos referentes a determinado conteúdo da química pura adequados ao processo de ensino aprendizagem do mesmo. Sua nomenclatura é Conhecimento Didático do Conteúdo, Pedagogical Content Knowledge - PCK e está dividido em três subdomínios: Conhecimento do Ensino de Química (Knowledge of Chemistry Teaching - KCT); Conhecimento das Características de Aprendizagem da Química (Knowledge of Features of Learning Chemistry - KFLC), e; Conhecimento dos Parâmetros da Aprendizagem de Química (Knowledge of Chemistry Learning Standards - KCLS).

\section{Conhecimento do Ensino de Química - KCT}

O Conhecimento do Ensino de Química - KCT considera o conhecimento do docente, mediante a potencialidade de determinada atividade perante o processo de aprendizagem; os recursos materiais, laboratoriais e virtuais, desde tabela periódica, modelos moleculares 3D, materiais laboratoriais, como vidrarias e equipamentos (pHmetro, capela, estufa, entre outros), até softwares (por exemplo, ChemDraw), aplicativos, sites ou vídeos.

Este subdomínio também considera o conhecimento de estratégias de ensino, por exemplo, como apresentar determinado conteúdo químico, incluindo desde estratégias básicas, como seminários, leitura, interpretação, discussão de texto e/ou síntese; a escolha de recursos, de experimentos laboratoriais, visitas técnicas, entrevistas ou aula de campo; inclusive, se necessário, o docente pode considerar a interdisciplinaridade da química em suas aplicações, como estratégia de ensino de química.

\section{Conhecimento das Características de Aprendizagem da Química - KFLC}

O subdomínio Conhecimento das Características de Aprendizagem da Química - KFLC considera conhecimentos relativos ao processo de assimilação, erros comuns e dificuldades, de modo que o conteúdo químico seja o objeto de aprendizagem e não o aluno, porém, faz-se necessário considerar a relação aluno-química, química como objeto, independentemente da particularidade deste estudante. Este subdomínio considera a forma como o aluno aprende 
química, qual é o interesse, curiosidade e expectativa deste discente quanto a determinada área química, o que pode ser considerado um elemento facilitador ou o que pode bloquear o conhecimento da química, ou mesmo, em determinada área química.

Os conhecimentos relativos a este subdomínio comumente estão associados ao impacto da estratégia de ensino, uma vez que o interesse, a assimilação e a expectativa do estudante oscilam, conforme a estratégia, o desenvolvimento e a avaliação, principalmente para a química, uma vez que, além das atividades lúdicas, pode-se realizar atividades experimentais.

\section{Conhecimento dos Parâmetros da Aprendizagem de Química - KCLS}

O Conhecimento dos Parâmetros da Aprendizagem de Química - KCLS inclui conhecimentos relativos aos parâmetros curriculares, à sequência dos conteúdos, à expectativa do professor com relação a determinada aprendizagem em determinado nível escolar, ou seja, que espera que o estudante tenha conhecimento ao concluir determinado nível escolar, e a meta quanto ao desenvolvimento de determinado conteúdo químico.

Vale ressaltar que o Ministério da Educação, através da Base Nacional Comum Curricular - BNCC estabelece habilidades mínimas necessárias para cada nível escolar, entretanto é a instituição de ensino que desenvolve a ementa das disciplinas conforme o nível escolar, atendendo tais habilidades, mas cabe ao docente a escolha do sequenciamento dos conteúdos, o conhecimento da natureza e conexões da química, assim como as habilidades e competências relativas a cada nível e a cada conteúdo, atentando-se quanto à interdisciplinaridade da química, principalmente com sequenciamento de conceitos relativos à matemática, física e biologia, necessários ao desenvolvimento do conteúdo químico.

\subsection{Crenças}

O CTSK considera duas crenças: a relativa à química pura, ou seja, ao domínio Conhecimento Químico, com nomenclatura Sobre Química e a relativa ao ensino de química, isto é, ao domínio Conhecimento Didático do Conteúdo, com nomenclatura Sobre Ensino e Aprendizagem da Química, e as crenças consideradas são tanto dos professores quanto dos alunos. 


\section{CONSIDERAÇÕES}

Os resultados obtidos atenderam ao objetivo desta pesquisa, em propor um modelo teórico capaz de descrever o conjunto de Conhecimento Especializado de Professores de Química, denominado CTSK. O qual poderá ser utilizado como ferramenta analítica nas diversas áreas da química, a partir das características delineadas.

Os resultados foram satisfatórios, sugere-se como estudos futuros a aplicação deste modelo como ferramenta analítica, algo que inclusive já está em andamento no grupo de pesquisa para as áreas de fisico-química e orgânica. Assim como, sugere-se estudar a proposta de categorias para o CTSK, seguindo a linha de desenvolvimento do MTSK.

\section{AGRADECIMENTOS}

Agradecemos ao IFMT - Campi Cuiabá, Cuiabá Bela Vista e São Vicente, e; ao Programa de Mestrado em Ensino do IFMT.

\section{REFERÊNCIAS}

BALL, D. L.; THAMES, M. H.; PHELPS, G. Content Knowledge for Teaching: What Makes It Special? Journal of teacher education, SAGE, New York, USA, v. 59, n. 5, p. 389-407, 2008.

BOGDAN, R.; BIKLEN, S. Investigação qualitativa em Educação: fundamentos, métodos e técnicas. Porto, Portugal: Porto Editora, 1994, p. 47-51

CARRILLO, J.; AVILA, D. I. E.; MORA, D. V.; MEDRANO, E. F. Un marco teórico para el conocimiento especializado del profesor de matemáticas. Huelva, Espanha: Universidad de Huelva Publicaciones, 2014.

CARRILLO YAÑEZ, J.; CLIMENT, N.; MONTES, M.; CONTRERAS, L. C.; FLORESMEDRANO, E.; ESCUDERO-ÁVILA, D.; VASCO, D.; ROJAS, N.; FLORES, P.; AGUILARGONZÁLEZ, Á.; RIBEIRO, M.; MUÑOZ-CATALÁN, M. C.

The mathematics teacher's specialised knowledge (MTSK) model. Research

in Mathematics Education, Taylor \& Francis, London, UK, p. 1-18, 2018.

CARRILLO YAÑEZ, J.; GONZÁLEZ, L. C. C.; NAVARRO, M. A. M. Reflexionando sobre el conocimiento del professor: Actas de las II Jornadas del Seminario de Investigación de Didactica de la Matemática de la Universidad de Huelva. 15 y 16 de Septiembre 2015. Huelva, Espanha, 2015. 106 p.

CORREIA, F. S. C. et al. O Estudo da Química no Cotidiano: As dificuldades para os alunos no ensino de Química. Ensino médio em diálogo, UFF, Niterói, 2015. Disponível em: 
http://www.emdialogo.uff.br/content/o-estudo-da-quimica-no-cotidiano-dificuldades-para-osalunos-no-ensino-de-quimica>. Acesso: 13 abr. 2017.

FERNANDEZ, C. Revisitando a base de conhecimentos e o conhecimento pedagógico do conteúdo (PCK) de professores de ciências. Revista Ensaio: Avaliação e Políticas Públicas em Educação, CESGRANRIO, Rio de Janeiro, v. 17, n. 2, p. 500-528, maio-ago 2015.

GARCIA, I. T. S. Implantação das Diretrizes Curriculares Nacionais para Formação de Professores de Química em uma Instituição Federal de Ensino Superior: Desafios e Perspectivas. Química Nova, SBQ, São Paulo, v. 32, n. 8, p. 2218-2224, 2009.

GIL, A. C. Como elaborar projetos de pesquisa. 4. ed. São Paulo: Atlas, 2002. 175 p.

GOES, Luciane F. de. Conhecimento Pedagógico do conteúdo: estado da arte no campo da educação e no ensino de química. Dissertação (Mestrado em Ensino de Química) - Universidade de São Paulo - USP, 2014. 155 p.

LIMA, S. S. Conhecimento especializado de professores de física: uma proposta de modelo teórico. Dissertação (Mestrado em Ensino) - Programa de Pós-Graduação Stricto Sensu em Ensino, Instituto Federal de Educação, Ciência e Tecnologia de Mato Grosso - IFMT, Cuiabá, 2018. $293 \mathrm{f}$.

LIMA, S. S.; COSTA, L. D.; SOARES, S. T. C.; SILVA FILHO, V. P.; MORIEL JUNIOR, J. G.; MELLO, G. J. Análise de PaP-eRs como primeira aproximação metodológica para configurar o modelo de conhecimento especializado de professores de física (PTSK). CONGRESSO INTERNACIONAL DE FORMAÇÃO E DESENVOLVIMENTO PROFISSIONAL DOCENTE - RESIDÊNCIA DOCENTE: PARADIGMA DE INTEGRAÇÃO TEORIA-PRÁTICA, 3., Cuiabá. Anais... p. 1 - 5. 2017.

LOPES, A. C. Discursos Curriculares na Disciplina Escolar Química. Ciência \& Educação, UNESP, Bauru, v. 11, n. 2, p. 263-278, 2005.

LOPES, A. R. C. Conhecimento Escolar em Química - Processo de Mediação Didática da Ciência. Química Nova na Escola, SBQ, São Paulo, n. 5, Maio 1997.

LUÍS, M.; MONTEIRO, R.; CARRILLO, J. Conhecimento Especializado do Professor para Ensinar Ciências. In: ENCONTRO NACIONAL DE EDUCAÇÃO EM CIÊNCIAS, XVI., 2015, Lisboa, Portugal. Anais... . Lisboa: APEduC, 2015. v. 1, p. 1 - 6.

MARQUES, M.; MORIEL JUNIOR, J. G. Conhecimento Especializado de Professores de Biologia: uma Análise de Pap-eR sobre Embriologia Humana. In: CONGRESO IBEROAMERICANO SOBRE CONOCIMIENTO ESPECIALIZADO DEL PROFESOR DE MATEMÁTICAS. Huelva: CGSE. 2019.

. Conhecimentos Especializados de Professor de Biologia Mobilizados em uma Aula Prática Sobre Interações Ecológicas. Revista REAMEC, Cuiabá (MT), v. 8, n. 2, p. 253-271, Maio-Ago, 2020. 
MASSENA, E. P; GUZZI FILHO, N. J; LUCIANA, P. SÁ. Produção de Casos para o Ensino de Química: Uma Experiência na Formação Inicial de Professores. Quimica Nova, SBQ, São Paulo, v. 36, n. 7, p. 1066-1072, 2013.

MELO, M.R; NETO, E.G.L. Dificuldades de Ensino e Aprendizagem dos Modelos Atômicos em Química. Química Nova na Escola, SBQ, São Paulo, v. 35, n. 2, p. 112-122, Maio. 2013.

MONTES, M.; CONTRERAS-GONZALEZ, L.; CARRILLO, J. Conocimiento del profesor de matemáticas: Enfoques del MKT y del MTSK. Simposio de la SEIEM, Bilbao, España, v. 1, 2013.

MORIEL JUNIOR, J. G. Conhecimento especializado para ensinar divisão de frações. Tese (Doutorado em Educação em Ciências e Matemática) - Programa de Pós-Graduação em Educação em Ciências e Matemática - PPGECEM, Rede Amazônica de Educação em Ciências e Matemática - REAMEC, Universidade Federal de Mato Grosso - UFMT, Cuiabá, 2014. 162 p.

; WIELEWSKI, G. D. Base de Conhecimento de Professores de Matemática: do Genérico ao Especializado. Rev. Ens. Educ. Cienc. Human., v. 18, n. 2, p.126-133, 2017.

OLIVEIRA, I; REZENDE, F. Discurso de Estudantes e Habitus Pedagógico em Cursos de Graduação em Ciências Naturais. Revista Brasileira de Pesquisa em Educação em Ciências RBPEC, UFMG, Belo Horizonte, v. 11, n. 3, 2011.

PETRUCCI, M. I. F. S. R. ; SCHNETZLER., R. P. Sobre a importância do conceito transformação química no processo de aquisição do conhecimento químico. Química Nova na Escola, SBQ, São Paulo, n. 8, nov. 1998.

QUADROS, A. L; SILVA, D. C.; ANDRADE, F. P; ALEME, H. G; RODRIGUES, S. O; SILVA, G. F. Ensinar e aprender Química: a percepção dos professores do Ensino Médio. Educar em Revista, UFPR, Curitiba, n. 40, p. 159-176, abr./jun. 2011.

ROQUE, N. F; SILVA, J. L P. B. A Linguagem Química e o Ensino da Química Orgânica. Química Nova, SBQ, São Paulo, v. 31, n. 4, p. 921-923, 2008.

SCHNETZLER, R. P. A pesquisa em ensino de química no Brasil: conquistas e perspectivas. Química Nova, SBQ, São Paulo, v. 25, n. Supl. 1, p. 14-24, 2002.

Construção do Conhecimento e Ensino de Ciências - O Modelo Transmissão-Recepção e o Ensino de Ciências. Em Aberto, MEC, Brasília, ano 11, n. 55, jul./set. 1992.

SHULMAN, L. S. Artigos. Professor of Education Emeritus, Stanford University, California, USA. Disponível em: <https://scholar.google.com.br/citations?user=QRW-VLcAAAAJ\&hl=ptBR>. Acesso em: 20 abr. 2020.

Knowledge and teaching: Foundations of the new reform. Harvard Educational Review, Cambridge, MA, USA, p. 1-22, Feb. 1987.

Those who understand: Knowledge growth in teaching. Education Researcher, SAGE, California, USA, Feb. 1986: 4-14. 
SIDM. Seminario de Investigación en Didáctica de la Matemática. Categorías de los subdomínios del MTSK (documento interno). Huelva, Espanha, 2016. 1 p. [Não publicado].

SILVA, A. A. da. A Construção do Conhecimento Científico no Ensino de Química. Revista Thema, IFSUL, Pelotas, v. 09, n. 02, 2012.

SILVA, R. M. G; SCHNETZLER, R. P. Constituição de professores universitários de disciplinas sobre ensino de Química. Química Nova, SBQ, São Paulo, v. 28, n. 6, São Paulo Nov./Dec. 2005.

SILVA, S. M.; EICHLER, M. L.; DEL PINO, J. C. As percepções dos professores de química geral sobre a seleção e a organização conceitual em sua disciplina. Química nova, SBQ, São Paulo, v. 26, n. 4, p. 585-594, 2003.

SOARES, S. T. C. Conhecimento Especializado de Professores de Química - CTSK:

Proposta de Modelo Teórico. 2019, 113f. Dissertação (Mestrado em Ensino) - Programa de Pós-Graduação Stricto Sensu em Ensino, Instituto Federal de Educação, Ciência e Tecnologia de Mato Grosso (IFMT), Cuiabá, 2019.

; LIMA, S. S.; CARBO, L.; MELLO, G. J. Aplicação da Metodologia PaP-eR para transposição do MTSK para diferentes áreas das Ciências da Natureza. In: CONGRESO IBEROAMERICANO SOBRE CONOCIMIENTO ESPECIALIZADO DEL PROFESOR DE MATEMÁTICAS. Huelva: CGSE. 2019.

VASCO, D.; MORIEL JUNIOR, J.; CONTRERAS, L. C. Subdominios del mathematics teacher's specialised knowledge (MTSK): KoT y KSM: definición, categorías y ejemplos. JORNADAS DE INVESTIGACIÓN EM DIDÁTICA DE LAS MATEMÁTICAS, 3., 2017, Huelva, Espanha. Anais. p. 29-37, 2017.

Submetido em: 25 de abril de 2020 .

Aprovado em: 18 de maio de 2020. 